\title{
Impact of pneumococcal conjugate vaccines \\ introduction on antibiotic resistance of Streptococcus pneumoniae meningitis in children aged 5 years or younger, Israel, 2004 to 2016
}

Shalom Ben-Shimol ${ }^{1,2}$, Noga Givon-Lavi ${ }^{1,2}$, David Greenberg ${ }^{1,2}$, Michal Stein ${ }^{3}$, Orli Megged ${ }^{4}$, Avihu Bar-Yochai ${ }^{5}$, Shahar Negari ${ }^{1,2}$, Ron Dagan ${ }^{2}$, on behalf of the Israel Bacteremia and Meningitis Active Surveillance Group ${ }^{6}$

1. The Pediatric Infectious Disease Unit, Soroka University Medical Center, Beer-Sheva, Israel

2. The Faculty of Health Sciences, Ben-Gurion University of the Negev, Beer-Sheva, Israel

3. Infectious Diseases and Infection Control Unit, Hillel Yaffe Medical Center, Hadera, Israel and Rappaport Faculty of Medicine, Technion - Israel Institute of Technology, Haifa, Israel

4. Pediatric Infectious Diseases Unit, Shaare Zedek Medical Center, affiliated with Hebrew University-Hadassah School of Medicine, Jerusalem, Israel

5. Infectious Disease Unit, Assaf Harofe Medical Center, Zerifin, Israel

6. Members of the Israel Bacteremia and Meningitis Active Surveillance Group have been cknowledged at the end of this article

Correspondence: Ron Dagan (rdagan@bgu.ac.il)

Citation style for this article:

Ben-Shimol Shalom, Givon-Lavi Noga, Greenberg David, Stein Michal, Megged Orli, Bar-Yochai Avihu, Negari Shahar, Dagan Ron, on behalf of the Israel Bacteremia and Meningitis Active Surveillance Group. Impact of pneumococcal conjugate vaccines introduction on antibiotic resistance of Streptococcus pneumoniae meningitis in children aged 5 years or younger, Israel, 2004 to 2016 . Euro Surveill. 2018;23(47):pii=1800081. https://doi.org/10.2807/1560-7917. ES.2018.23.47.1800081

Article submitted on 21 Feb 2018 / accepted on 09 Jul 2018 / published on 22 Nov 2018

Background: Empiric treatment of pneumococcal meningitis includes ceftriaxone with vancomycin to overcome ceftriaxone resistant disease. The addition of vancomycin bears a risk of adverse events, including increased antibiotic resistance. We assessed antibiotic resistance rates in pneumococcal meningitis before and after pneumococcal conjugate vaccine (PCV) implementation. Methods: All pneumococcal meningitis episodes in children aged 5 years and younger, from 2004 to 2016, were extracted from the nationwide bacteremia and meningitis surveillance database. For comparison purposes, we defined pre-PCV period as 2004-2008 and PCV13 period as 2014-2016. Minimal inhibitory concentration (MIC)>0.06 and > $0.5 \mu \mathrm{g} /$ $\mathrm{mL}$ were defined as penicillin and ceftriaxone resistance, respectively. Results: Overall, 325 episodes were identified. Pneumococcal meningitis incidence rates declined non-significantly by $17 \%$, comparing PCV13 and pre-PCV periods. Throughout the study, $90 \%$ of isolates were tested for antibiotic susceptibility, with $26.6 \%, 2.1 \%$ and $0 \%$ of isolates resistant to penicillin, ceftriaxone and vancomycin, respectively. Mean proportions ( \pm SD) of meningitis caused by penicillin-resistant pneumococci were $40.5 \% \pm 8.0 \%$ and $9.6 \% \pm 7.4 \%$ in the pre-PCV and the PCV 13 periods, respectively, resulting in an overall $83.9 \%$ reduction (odd ratio:0.161; 95\% confidence interval: $0.059-$ 0.441 ) in penicillin resistance rates. The proportions of meningitis caused by ceftriaxone resistant pneumococci were $5.0 \% \pm 0.8 \%$ in the pre-PCV period, but no ceftriaxone resistant isolates were identified since 2010. Conclusions: $\mathrm{PCV}_{7} / \mathrm{PCV}_{13}$ sequential introduction resulted in> $80 \%$ reduction of penicillin- resistant pneumococcal meningitis and complete disappearance of ceftriaxone resistant disease. These trends should be considered by the treating physician when choosing an empiric treatment for pneumococcal meningitis.

\section{Introduction}

Bacterial meningitis is a major cause of morbidity and mortality in children worldwide, with Streptococcus pneumoniae being the leading cause of bacterial meningitis in up to $60 \%$ of cases $[1,2]$. Pneumococcal meningitis has high rates of long-term complications (e.g. behavioural/intellectual disorders, hearing loss and neurologic deficits), with mortality rates of up to $26 \%$ [2].

The early and rapid administration of antibiotics is crucial to increase survival and reduce morbidity in pneumococcal meningitis and the choice of empiric antibiotics should be based on the local epidemiology of antibiotic susceptibility, among other factors [3]. Antibiotic resistance definitions for pneumococcal meningitis are stricter than those for non-meningitis pneumococcal infections, with minimal inhibitory concentrations (MIC) cut-offs of $>0.06 \mu \mathrm{g} / \mathrm{mL}$ for penicillin resistance and $\mathrm{MIC}>0.5 \mu \mathrm{g} / \mathrm{mL}$ for ceftriaxone resistance, according to the Clinical and Laboratory Standards Institute (CLSI) [2,4]. 


\section{TABLE 1}

Number of pneumococcal meningitis episodes and incidence rates in children aged 5 years or younger, Israel, July 2000-June 2016

\begin{tabular}{|c|c|c|}
\hline \multirow{2}{*}{ Time period } & \multicolumn{2}{|c|}{ Pneumococcal meningitis } \\
\hline & Number of episodes & Incidence per 100,000 \\
\hline July oo-June 01 & 26 & 3.98 \\
\hline July 01-June 02 & 30 & 4.49 \\
\hline July $02-J u n e ~ 03$ & 48 & 7.05 \\
\hline July 03-June 04 & 26 & 3.75 \\
\hline July $04-J$ une $05^{a}$ & 25 & 3.55 \\
\hline July 05 -June $06^{a}$ & 27 & 3.78 \\
\hline July o6-June $07^{\text {a }}$ & 33 & 4.55 \\
\hline July o7-June $08^{\text {a }}$ & 27 & 3.66 \\
\hline July 08-June o9 & 38 & 5.08 \\
\hline July 09-June 10 & 29 & 3.80 \\
\hline July 10 -June 11 & 23 & 2.93 \\
\hline July $11-J u n e ~ 12$ & 27 & 3.36 \\
\hline July 12 -June 13 & 13 & 1.58 \\
\hline July 13 -June 14 & 27 & 3.21 \\
\hline July 14 -June $15^{b}$ & 28 & 3.27 \\
\hline July 15 -June $16^{b}$ & 28 & 3.21 \\
\hline \multicolumn{3}{|l|}{ IRR $(95 \% \mathrm{CI})$} \\
\hline PCV 13 vs pre-PCV & NA & $0.83(0.60-1.15)$ \\
\hline
\end{tabular}

$\mathrm{Cl}$ : confidence interval; IRR: incidence rate ratio; NA: not available; PCV: pneumococcal conjugate vaccine.

a Period termed as 'Pre-PCV period' defined as 2004-2008.

b Period termed as 'PCV 13 period' defined as 2014-2016.

Before the introduction of pneumococcal conjugate vaccines (PCVs) worldwide, an increase in pneumococcal antimicrobial (mainly penicillin) resistance had been observed [5-8], resulting in modifications of the empiric management of meningitis [8,9]. Initially, third generation cephalosporins (cefotaxime and ceftriaxone) became the standard therapy in children and subsequently, in the mid-1990s, reports of cephalosporin-resistant pneumococcal meningitis led to the recommendation of adding vancomycin as empiric therapy in suspected cases $[3,10]$. Therefore, the empiric treatment in cases of suspected pneumococcal meningitis currently includes intra-venous ceftriaxone and vancomycin pending culture results [10-12].

Due to its low penetration through the meninges, vancomycin is given at high doses $(60 \mathrm{mg} / \mathrm{kg} / \mathrm{day})$. The addition of empiric treatment with vancomycin may rarely result in side effects, such as hypersensitivity, nephrotoxicity (especially at high doses used to treat pneumococcal meningitis) and hearing loss $[2,13]$. Furthermore, using vancomycin increases the selection and exposure of bacteria to antibiotics and may accelerate the emergence of strains resistant to vancomycin, which is one of the antibiotics saved as last-resort treatment against bacteria resistant to other antimicrobial drugs $[2,13-15]$.
In July 2009, the 7-valent pneumococcal conjugate vaccine $\left(\mathrm{PCV}_{7}\right)$ was introduced to the Israeli national immunisation programme (NIP) and in November 2010, was replaced by the 13-valent pneumococcal conjugate vaccine (PCV13) [16]. By December 2012, the proportion of children who received two or more doses of $\mathrm{PCV}_{7}$ and PCV13 was greater than 95\% [16]. Following PCV7/ PCV13 sequential introduction, rates of pneumococcal meningitis and other invasive pneumococcal disease (IPD) caused by vaccine-serotype pneumococci substantially declined by ca $95 \%$ in children aged 5 years or younger [17]. In contrast, overall pneumococcal meningitis rates were reduced non-significantly by $27 \%$ and remaining cases mainly reflected an increased rate of non-vaccine serotype pneumococcal meningitis [17]. Besides the impact observed worldwide on IPD rates caused by vaccine-serotypes, including meningitis, PCV introduction in the United States (US) was also followed by a reduction in antibiotic resistance rate $[18,19]$. This reduction may have derived from the elimination of resistant vaccine strains and the increase in non-vaccine strains $[17,18,20]$ that are more frequently antibiotic susceptible, as they were previously less exposed to antibiotic selection stress [18].

The empiric treatment of suspected pneumococcal meningitis with ceftriaxone and vancomycin both in Israel and in many settings worldwide $[2,11]$ was rarely revisited following PCVs introduction. Nevertheless, vancomycin treatment might not be justified, if ceftriaxone resistance rates are shown to be low [3].

We assessed antibiotic resistance rates in pneumococcal meningitis episodes before and after PCV introduction to the Israeli NIP, in an attempt to optimise current treatment recommendations.

\section{Methods}

The ongoing, prospective, nationwide, populationbased and active surveillance study, conducted by the Israeli Paediatric Bacteraemia and Meningitis Group, was initiated in 1989. The current report describes data spanning over a 12-year period (July 2004-June 2016). Incidence rates of pneumococcal meningitis are presented from July 2000 , to allow better appreciation of secular trends and fluctuations.

The study was approved by the Institutional Ethics Committees of the participating medical centres.

\section{Setting and study population}

The study population comprised all children aged 5 years or younger in Israel. As of 2016 , Israel had a population of ca 875,000 children in this age group [21].

The study has been conducted in all 26 medical health centres routinely obtaining cerebrospinal fluid (CSF) cultures from children $[16,22]$, no CSF cultures are obtained outside these centres, enabling us to collect the majority of culture-confirmed pneumococcal meningitis cases in Israel. 
Pneumococcal meningitis incidence rates in children aged 5 years and younger, Israel, July 2000-June 2016 (n = 455)

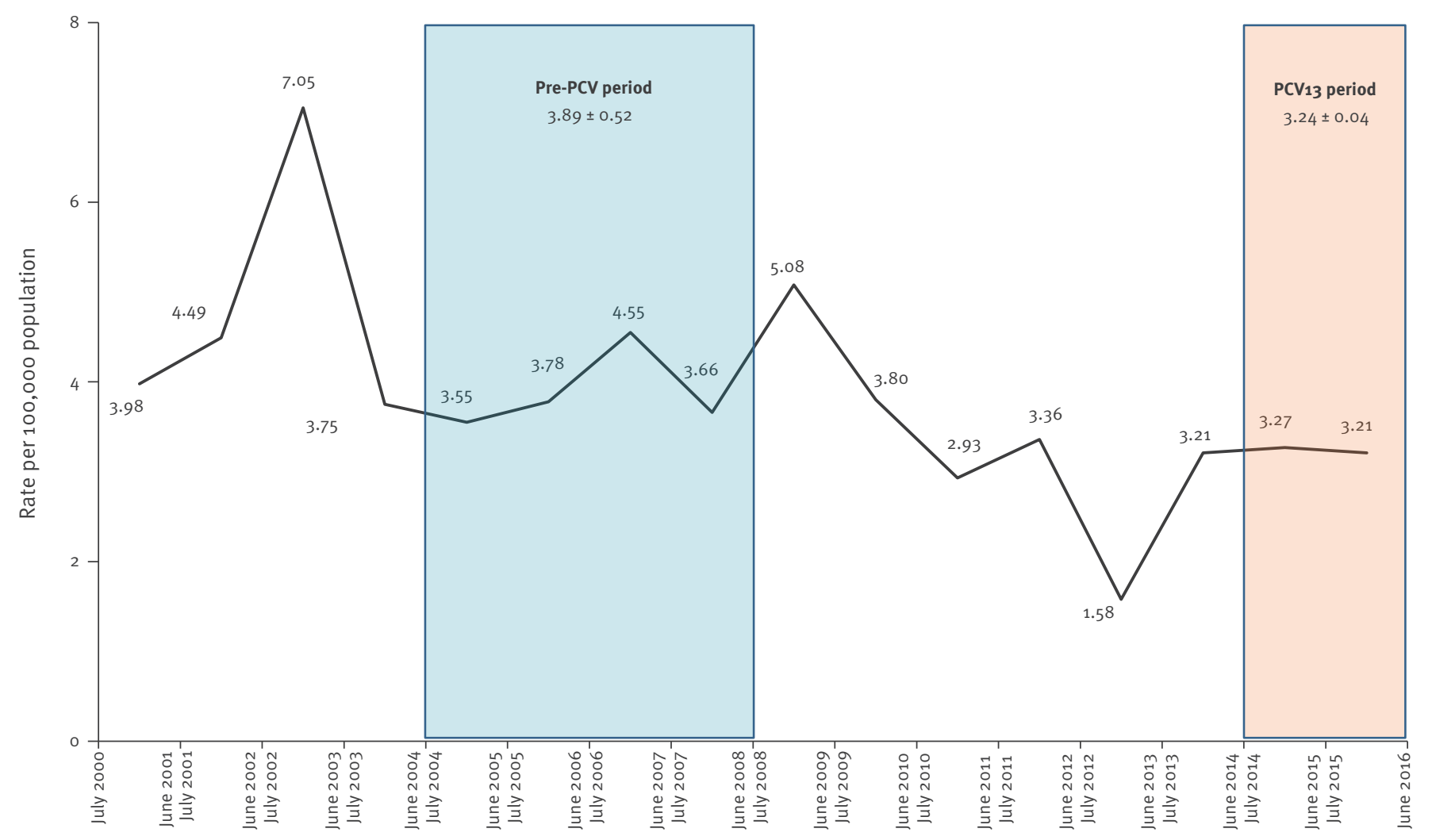

PCV: pneumococcal conjugate vaccine.

'Pre-PCV period' defined as 2004-2008 and 'PCV 13 period' defined as 2014-2016.

Incidence rates per 100,000 children.

Local investigators in each centre responded to a monthly distributed questionnaire sent by the principal investigator at the study headquarters.

\section{Case definitions}

A pneumococcal meningitis episode was defined as an illness during which $S$. pneumoniae was isolated from either CSF or from blood with laboratory signs suggestive of meningitis (e.g. CSF pleocytosis). Nonculture diagnoses (polymerase chain reaction, antigen testing, Gram stain results or clinical diagnosis) were excluded. In Israel, $<5 \%$ of all meningitis episodes are diagnosed by non-culture methods.

\section{Antibiotic susceptibility testing}

According to the National Committee for Clinical and Laboratory definitions for antibiotic susceptibility in pneumococcal meningitis, isolates with a MIC of> 0.06 $\mu \mathrm{g} / \mathrm{mL}$ for penicillin were determined as penicillin resistant. Isolates with an MIC>0.5 $\mu \mathrm{g} / \mathrm{mL}$ for ceftriaxone were defined as ceftriaxone resistant $[2,13]$.
Uptake of pneumococcal conjugate vaccines PCV7 NIP was initiated in July 2009 with a catch-up campaign in children aged 2 years or younger $[17,23]$. In November 2010, PCV13 replaced $\mathrm{PCV}_{7}$ without further catch-up.

Vaccine uptake evaluation methods were previously described [16,17]. By June 2011 and December 2012, ca $80 \%$ and ca $90 \%$, respectively, of 7-11 month old children received two or more doses of $\mathrm{PCV}_{7}$ and/or $\mathrm{PCV}_{13}$ and ca $95 \%$ received two or more $\mathrm{PCV}_{13}$ doses by June 2014 and June 2015.

By June 2011 and December 2012, 36\% and 87\%, respectively, of children aged 24-35 months, received three or more $\mathrm{PCV}_{7} / \mathrm{PCV}_{13}$ doses and $>90 \%$ received three or more doses of $\mathrm{PCV}_{13}$ by June 2014 and June 2015.

\section{Data analysis}

Annual (July to June) incidence rates were calculated as the number of CSF positive culture cases divided by the 
TABLE 2

Proportions of penicillin-resistant and ceftriaxone-resistant pneumococcal meningitis of all isolates in children aged 5 years and younger, Israel, July 2004-June 2016

\begin{tabular}{|c|c|c|c|c|}
\hline \multirow{2}{*}{ Time period } & \multicolumn{2}{|c|}{ Penicillin MIC > $0.06 \mu \mathrm{g} / \mathrm{mL}$} & \multicolumn{2}{|c|}{ Ceftriaxone MIC >0.5 $\mu \mathrm{g} / \mathrm{mL}$} \\
\hline & Number of isolates & Percent & Number of isolates & Percent \\
\hline July $04-J u n e 05^{\text {a }}$ & $7 / 22$ & 31.8 & $1 / 22$ & 4.5 \\
\hline July 05 -June $06^{\mathrm{a}}$ & $11 / 22$ & 50.0 & $1 / 20$ & 5.0 \\
\hline July 06 -June $07^{a}$ & $14 / 32$ & 43.8 & $2 / 33$ & 6.1 \\
\hline July 07-June $08^{a}$ & $8 / 22$ & 36.4 & $1 / 23$ & $4 \cdot 3$ \\
\hline July 08-June o9 & $9 / 34$ & 26.5 & $0 / 36$ & 0.0 \\
\hline July 09-June 10 & $10 / 23$ & 43.5 & $1 / 22$ & 4.5 \\
\hline July 10 -June 11 & $2 / 22$ & 9.1 & $0 / 22$ & 0.0 \\
\hline July $11-J u n e ~ 12$ & $5 / 25$ & 20.0 & $0 / 25$ & 0.0 \\
\hline July 12 -June 13 & $2 / 11$ & 18.2 & $0 / 11$ & 0.0 \\
\hline July 13 -June 14 & $4 / 26$ & 15.4 & $0 / 26$ & 0.0 \\
\hline July 14 -June $15^{b}$ & $1 / 23$ & $4 \cdot 3$ & $0 / 23$ & 0.0 \\
\hline July 15 -June $16^{b}$ & $4 / 27$ & 14.8 & $0 / 28$ & 0.0 \\
\hline \multicolumn{5}{|l|}{ OR $(95 \% \mathrm{Cl})$} \\
\hline PCV 13 vs pre PCV & NA & $0.16(0.06-0.44)$ & NA & NA \\
\hline
\end{tabular}

$\mathrm{Cl}$ : confidence interval; MIC: Minimal inhibitory concentration; NA: not available; OR: odds ratio; PCV: pneumococcal conjugate vaccine.

a Period termed as 'Pre-PCV period' defined as 2004-2008.

b Period termed as 'PCV 13 period' defined as 2014-2016.

total population at risk during each year of the study [21].

For episodes in which the serotype and/or serogroup were missing, a detailed extrapolation was conducted, as described elsewhere [22]. Briefly, the proportion of episodes attributed to each serotype-group (vaccine type and non-vaccine type) was assigned from the ageand ethnicity-specific strata, assuming serotype data were missing at random.

Since 2009-2010, the proportion of isolates with serotype determination increased to >95\% from ca $60 \%$ in the pre-PCV period (2004-2008) [22].

To assess changes in meningitis incidence and proportions of antibiotic resistance (penicillin MIC > 0.06 ; ceftriaxone MIC > 0.5) of all isolates, we used annual rates. Data are presented for all study years (20042016). In addition, we compared rates in the pre-PCV period (July 2004-June 2008) and the last two study years (July 2014-June 2016) as the PCV13 period.

Incidences were calculated using the birth cohorts born in Israel, according to the Israeli Central Bureau of Statistics reports [21]. Incidence rate ratios (IRRS) and $95 \%$ confidence intervals (Cls) were calculated for meningitis rates.

Proportions of antibiotic resistance of all isolates and odds ratios (ORs) with $95 \% \mathrm{Cl}$ were calculated. $\mathrm{P}$ values of dynamics in proportions of antibiotic resistance were calculated.
Data were analysed with SPSS 23.00 software. Univariate analyses were conducted using two-tailed chi-squared test or Student t-test, where appropriate. A p value $<0.05$ was considered statistically significant.

\section{Results}

During the study period, 325 pneumococcal meningitis episodes were identified in children aged 5 years or younger. The mean age was $14.5 \pm 13.7$ months, and median age was 10.1 months, with $54.8 \%(n=178)$ of children aged 12 months of less. Of the 325 episodes, $53.2 \%(n=173)$ were in males.

Of all 325 isolates, 289 (88.9\%) and 291 (89.5\%) were tested for penicillin and ceftriaxone susceptibility, respectively. Overall, throughout the study, 77 (26.6\%) isolates were penicillin-resistant and six (2.1\%) isolates were ceftriaxone-resistant. All $(n=290)$ tested isolates were susceptible to vancomycin.

\section{Pneumococcal meningitis rates dynamics}

Following PCV13 introduction, meningitis rates declined non-significantly by $17 \%$ (IRR: 0.83 ; 0.60-1.15) comparing the $\mathrm{PCV}_{13}$ and the pre-PCV periods (Table 1, Figure 1).

\section{Proportions of penicillin-resistant isolates}

In the pre-PCV period, proportions of meningitis caused by penicillin-resistant pneumococci were $40.5 \% \pm 8.0 \%$ (Table 2, Figure 2). In the $\mathrm{PCV}_{13}$ period, these proportions significantly declined by $83.9 \%(O R=0.16$; $0.06-$ 0.44 ) and were $9.6 \% \pm 7.4 \%$. Similarly, a significant declining trend of proportions of penicillin-resistant 


\section{FIGURE 2}

Proportions of penicillin-resistant pneumococcal meningitis of all isolates in children aged 5 years or younger, Israel, July 2004-June 2016 ( $\mathrm{n}=289$ )

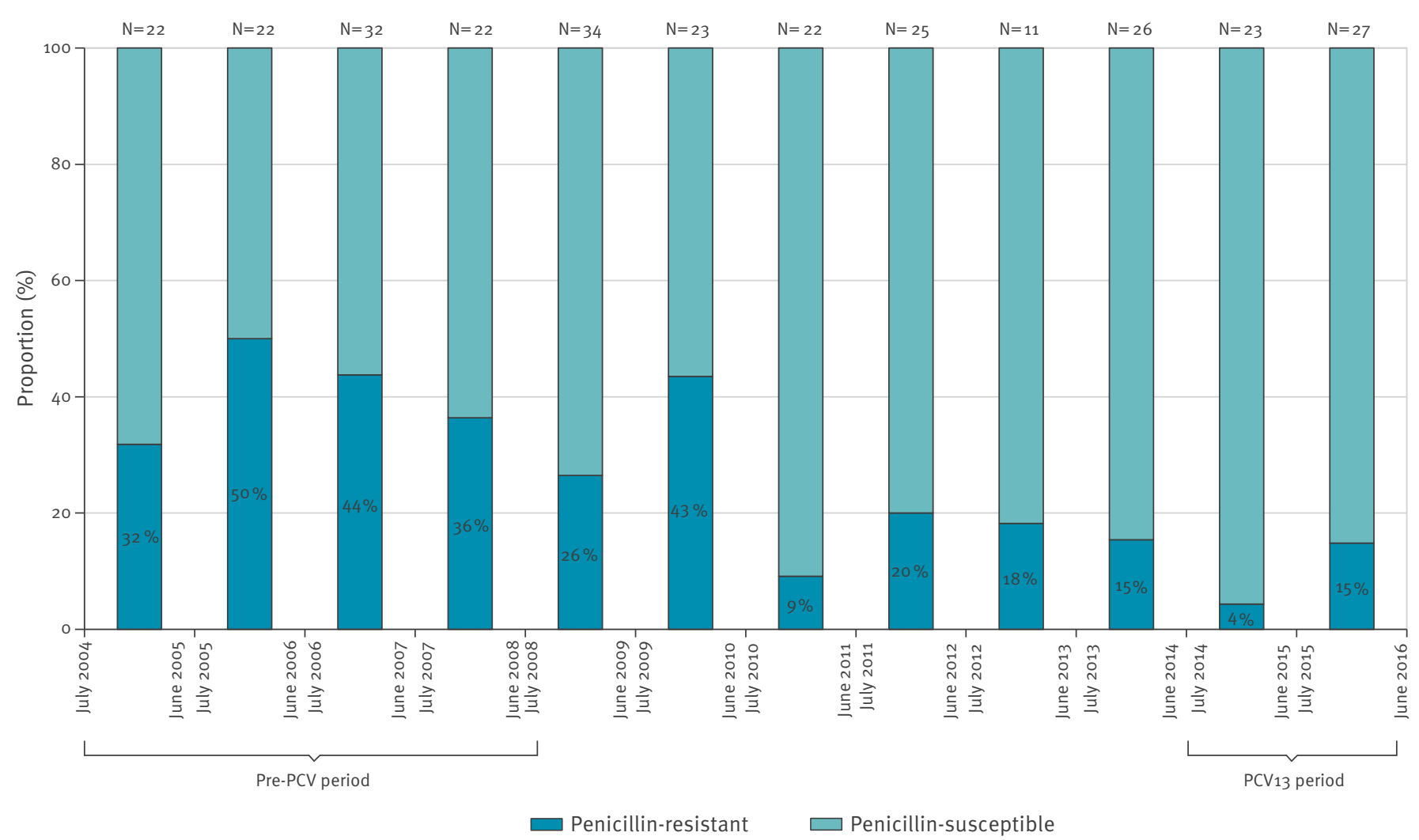

PCV: pneumococcal conjugate vaccine.

Penicillin-resistant pneumococcal meningitis = minimum inhibitory concentration (MIC) > $0.06 \mu \mathrm{g} / \mathrm{mL}$.

'Pre-PCV period' defined as 2004-2008 and 'PCV 13 period' defined as 2014-2016.

pneumococcal meningitis within pneumococcal isolates is presented in supplement.

\section{Proportions of ceftriaxone-resistant pneumococcal isolates in pneumococcal meningitis}

In the pre-PCV period, proportions of meningitis caused by ceftriaxone-resistant pneumococci were $5.0 \% \pm 0.8 \%$ (Table 2, Figure 3). Following PCV13 introduction, these proportions declined to $0.0 \%$. No cases of ceftriaxone resistant isolates were observed since July 2010.

Proportions of PCV13 serotypes (VT13), and non-VT13 pneumococcal isolates in antibioticresistant meningitis

Of 40 penicillin-resistant isolates in the pre-PCV period, 26 had a known serotype. Since July 2009, all 28 penicillin resistant isolates had known serotypes. Of 26 resistant isolates in the pre-PCV period, 21 were VT13, while in the $\mathrm{PCV}_{13}$ period, none $(0 / 5)$ of the resistant isolates were VT13 (Figure 4).
Within the non-VT13 group, penicillin resistance rates declined from $33.3 \%$ in the pre-PCV period to $10.4 \%$ in the PCV13 period $(p=0.049)$.

For the six ceftriaxone resistant isolates (all before July 2010), four isolates had a known serotype; all three isolates from the pre-PCV period were $\mathrm{VT}_{13}$ and the one isolate from the year 2009-2010 was non-VT13.

\section{Discussion}

The sequential introduction of $\mathrm{PCV}_{7} / \mathrm{PCV}_{13}$ into the Israel NIP resulted in an $80 \%$ decline in the incidence of antibiotic-resistant pneumococcal meningitis, even though the overall pneumococcal meningitis incidence rates did not decline significantly; reflecting the near elimination of disease caused by vaccine serotypes.

Before PCV implementation, vaccine-serotypes were the most successful serotypes in nasopharyngeal colonisation and therefore the most frequent pathogens causing pneumococcal diseases [24]. This frequent and relatively prolonged colonisation and involvement 


\section{FIGURE 3}

Proportions of ceftriaxone-resistant pneumococcal meningitis of all isolates in children aged 5 years and younger, Israel, July 2004-June $2016(n=291)$

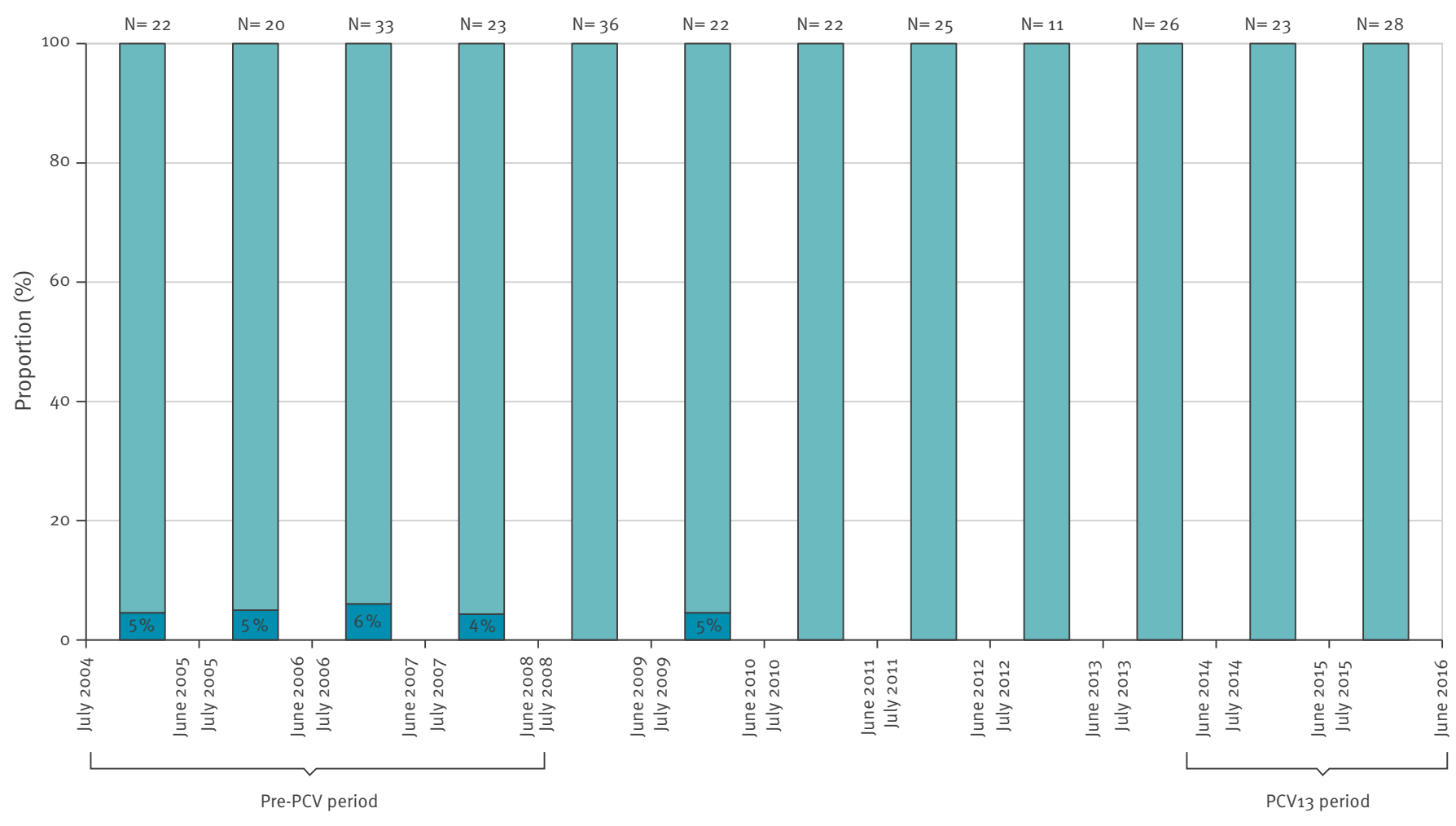

Ceftriaxone-resistant

Ceftriaxone-susceptible

PCV: pneumococcal conjugate vaccine.

Ceftriaxone-resistant pneumococcal meningitis = minimal inhibitory concentration $>0.5 \mu \mathrm{g} / \mathrm{mL}$.

'Pre-PCV period' defined as 2004-2008 and 'PCV 13' period defined as 2014-2016.

in disease resulted in continuous antibiotic pressure (selection for an antibiotic-resistant strain deriving from frequent or prolonged exposure to antibiotics) due to the high antibiotic consumption in young children and thus leading to the emergence of antibiotic resistance and multi-drug resistance [25].

In the pre-PCV era, an increased rate of antibiotic non-susceptibility in pneumococcal meningitis was observed in many sites worldwide $[5-8,13,14,26,27]$. This increase supports the well-known phenomenon of increased antibiotic resistance following the continuous usage of antibiotic, driven by selective pressure [24]. In southern Israel, seasonal variations in antibiotic resistance among otitis media pneumococcal isolates were associated with substantial variations in antibiotic consumption [28]. Furthermore, increased resistance to multiple antibiotics among nasopharyngeal carried pneumococcal isolates was associated with increased azithromycin consumption [29]. A recent study from southern Israel showed reduction in overall antibiotics dispatched prescription rates in children following $P C V_{7} / P_{13}$ sequential introduction [30], further supporting our hypothesis. Following $\mathrm{PCV}_{7}$ introduction, a substantial decrease in pneumococcal meningitis caused by $\mathrm{PCV}_{7}$ serotypes was observed, along with an increase in disease caused by nonvaccine serotypes, including strains non-susceptible to antibiotics $[12,18,19,31]$. Similarly, following PCV13 introduction, a substantial decline in $\mathrm{PCV}_{13}$-serotypes disease rates, along with an increase in non-VT13 serotypes were observed [17,23,32-34].

The reduction in disease caused by $\mathrm{VT}_{13}$ serotypes is accompanied by a reduction in the carriage of vaccine serotypes [24] resulting in decreased 'antibiotic pressure'. In the pre-PCV era, vaccine-serotypes were the main pneumococcal serotypes carried and responsible for causing disease; consequently, these were the main serotypes exposed to antibiotic pressure. It is not surprising, therefore, that these serotypes were the main strains exhibiting antibiotic resistance in the pre-PCV era. In contrast, non-vaccine serotypes were less exposed to antibiotic pressure and with the near elimination of vaccine-serotypes, the main burden of antibiotic resistance was eliminated. Nevertheless, it 
Proportions of PCV13 serotypes of all penicillin-resistant pneumococcal meningitis isolates in children aged 5 years or younger, Israel, July 2004-June $2016(\mathrm{n}=325)$

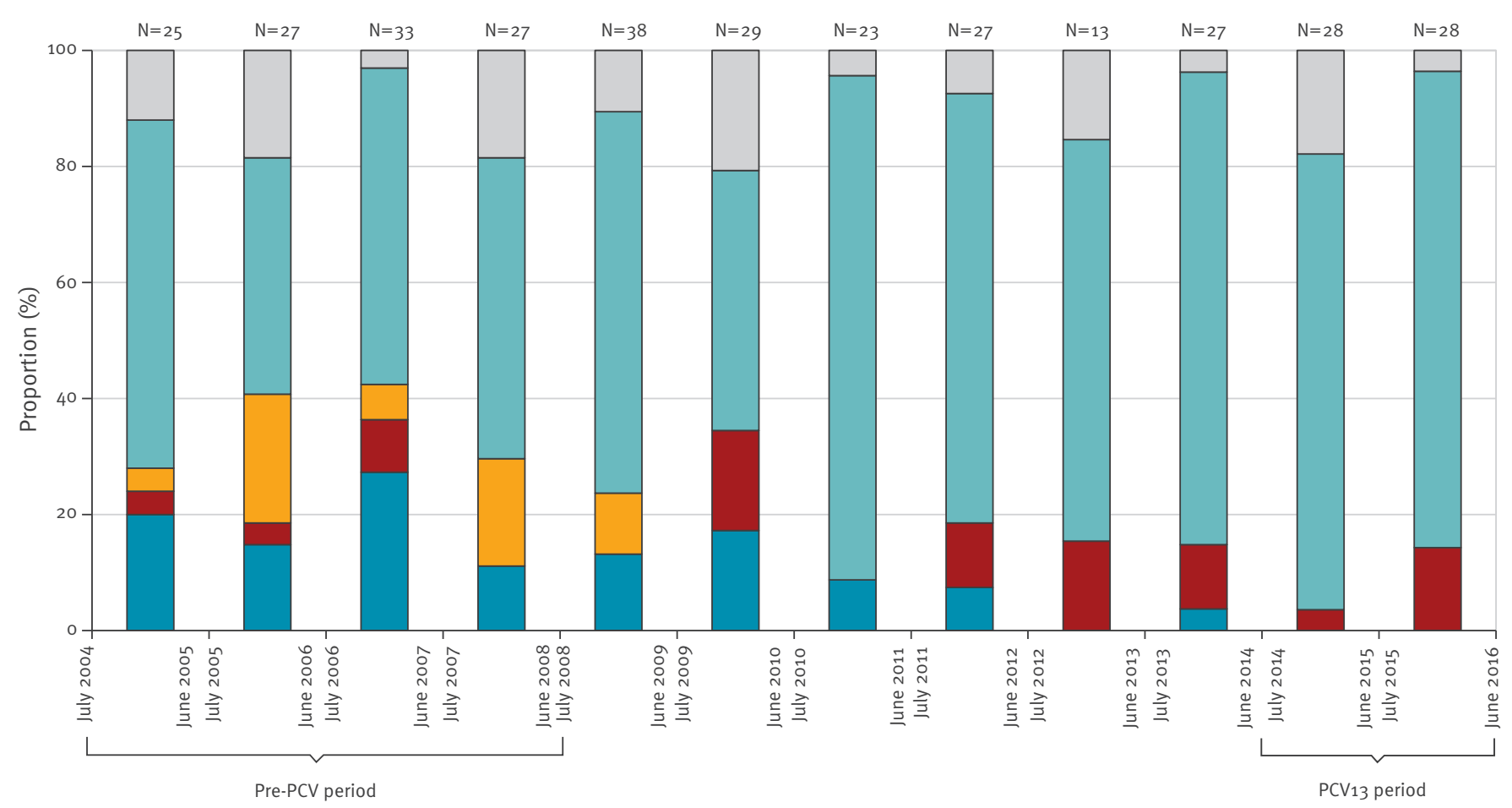

Pen-R VT13

Pen-R non-VT13

$\square$ Pen-R unknown serotype

$\square$ Penicillin-susceptible

$\square$ Not tested for AB-R

PCV: pneumococcal conjugate vaccine.

'Pre-PCV period' defined as 2004-2008 and 'PCV 13 period' defined as 2014-2016.

is important to recognise the possibility of new emerging antibiotic resistant strains among non-vaccine serotypes, deriving from the time elapsed since PCV13 introduction and increased exposure of the now predominating non-vaccine serotypes to antibiotic pressure. Indeed, following $\mathrm{PCV}_{7}$ introduction in the US, rapid replacement with penicillin-non-susceptible nonvaccine serotypes was observed [35]. However, when the overall impact on antibiotic resistance was evaluated, the outcome was positive.

Currently in Israel, treatment of pneumococcal meningitis in children involves empiric addition of vancomycin to ceftriaxone. It was previously suggested that in the epidemiologic setup of low ceftriaxone resistance ( $<1 \%$ ), it may be suitable to treat suspected pneumococcal meningitis cases empirically with ceftriaxone only, without adding vancomycin to the treatment regimen [3]. With this in mind, it may be suitable to recommend ceftriaxone only as an empiric treatment for pneumococcal meningitis in Israel (and possibly other settings where ceftriaxone resistance is low) assuming there is suitable ongoing surveillance. A possible advantage to removing vancomycin from the empiric treatment of meningitis would be in the reduction of potential side effects to the drug, including the emergence of strains resistant to vancomycin.

Continuous surveillance is needed to better understand and track antibiotic resistance rates, as well as identifying emerging resistant serotypes. In addition, surveillance could help to possibly developing new, broader (higher valency or protein based), pneumococcal vaccines.

The major strengths of our study include the utilisation of prospective, active and population-based methodology, as well as a large number of cases. The relatively long time period elapsed since PCV13 introduction to the Israeli NIP (6 years) also enables a more accurate evaluation of PCV13 impact. The main limitation of our study is the relatively high rate of undetermined serotypes early in the pre-PCV period. In recent years, however, the rate of extrapolated serotypes has dropped to $<5 \%$ of all isolated pneumococci. Notably, while in the pre-PCV period only $65 \%$ of penicillin-resistant isolates had a known serotype, this proportion decreased to $0 \%$ and has remained so since July 2009. However, while incomplete data on serotypes is a limitation, testing of penicillin resistance was done for ca $90 \%$ of 
isolates providing a comprehensive picture of penicillin resistance dynamics.

An additional limitation lies in the analysis of IRRs and ORs comparing grouped study years according to PCVs uptake (i.e. pre-PCV, $\mathrm{PCV}_{7}$ and $\mathrm{PCV}_{13}$ period) and the relatively small sample size in each year. Nevertheless, incidence rates for overall pneumococcal meningitis and antibiotic resistance proportions among all isolates and serotype sub-groups (VT13, non-VT13) are presented for each individual year. Additionally, chisquare for linear trend was calculated for penicillin resistance rates (proportions) and showed statistically significant reduction.

In summary, $\mathrm{PCV}_{7} / \mathrm{PCV}_{13}$ sequential introduction resulted in $>80 \%$ reduction of penicillin-resistant incidence rates of pneumococcal meningitis, in parallel with the disappearance of ceftriaxone resistant disease in Israeli children aged 5 years or younger. These trends should be considered by physicians when choosing an empiric treatment for pneumococcal meningitis.

\section{Acknowledgements}

The Israeli paediatric bacteraemia and meningitis group: Ron Dagan, principal investigator, Beer Sheva; Jacob Amir, Petah-Tikva; Galia Barkai, Tel Hashomer; Diana Averbuch, Jerusalem; Shraga Aviner, Ashkelon; Ahuva Bachinski, Hadera; Avihu Bar-Yochai, Zerifin; Ilana Benedikt, Jerusalem; Rita Bernstein, Rehovot; Tal Brosh-Nissimov, Ashdod; Nael Elias, Nazareth; Dan Engelhard, Jerusalem; Moshe Ephros, Haifa; Daniel Glikman, Nahariya; Giora Gottesman, KfarSaba; Galia Grisaru-Soen, Tel-Aviv; Alex Guri, Rehovot; Imad Kassis, Haifa; Nathan Keller, Tel Hashomer; Zina Korenman, Jerusalem; Hannah Leskes, Ashdod; Anthony Luder, Safed; Orli Megged, Jerusalem; Dan Miron, Afula; Meirav Mor, Petah-Tikva; Avi Peretz, Tveria; Uri Rubinstein, Netania; Yechiel Schlesinger, Jerusalem; David Schwartz, Tel-Aviv; Itamar Shalit, Petach Tikva; Eli Somekh, Holon; Isaac Srugo, Haifa; Michal Stein, Hadera; Yonatan Yeshayahu, Ashdod; Alvira Zbriger, Hadera; Miriam Zucker, Zfat.

The study was funded in part by Pfizer. The current study was conducted as a project with grant no. $0887 \mathrm{X}_{1-4603}$ from Pfizer. It was also was part of the projects conducted by the Israeli bacteraemia and Meningitis Active Surveillance Group which has been conducting nationwide surveillance on invasive diseases on a voluntary base since 1989. The funding body did not play any role in any of the stages of the manuscript preparation.

\section{Conflict of interest}

S. Ben-Shimol: Grant, Speaker, Pfizer; D. Greenberg: Grants, consulting, speaker, MSD; consulting, speaker, Pfizer; R. Dagan: Grants, consulting, speaker, Pfizer; grant, consulting, MSD; consulting, MeMed.

\section{Authors' contributions}

Conception and design: Shalom Ben-Shimol, David Greenberg, Ron Dagan; Review of the literature: Shalom Ben-Shimol, Ron Dagan; Acquisition of data: Noga GivonLavi, David Greenberg, Michal Stein, Orli Megged, Avihu
Bar-Yochai, Ron Dagan; Analysis and interpretation of data: Shalom Ben-Shimol, Noga Givon-Lavi, David Greenberg, Michal Stein, Orli Megged, Avihu Bar-Yochai, Shahar Negari, Ron Dagan; Drafting the article: Shalom Ben-Shimol Noga Givon-Lavi, David Greenberg, Michal Stein, Orli Megged, Avihu Bar-Yochai, Shahar Negari, Ron Dagan; Revising the article critically for important intellectual content: Shalom Ben-Shimol, Noga Givon-Lavi David Greenberg, Michal Stein, Orli Megged, Avihu Bar-Yochai, Shahar Negari, Ron Dagan; Statistical analysis: Shalom Ben-Shimol, Noga Givon-Lavi, David Greenberg, Ron Dagan; Final approval of the submitted version: Shalom Ben-Shimol, Noga Givon-Lavi, David Greenberg, Michal Stein, Orli Megged, Avihu Bar-Yochai, Shahar Negari, Ron Dagan; Each member of the collective group is in charge of the data collection in their respective site.

\section{References}

1. O’Brien KL, Wolfson LJ, Watt JP, Henkle E, Deloria-Knoll M, McCall N, et al. Burden of disease caused by Streptococcus pneumoniae in children younger than 5 years: global estimates. Lancet. 2009;374(9693):893-902. https://doi. org/10.1016/S0140-6736(og)61204-6 PMID: 19748398

2. Allan T. van de beek D, Michael S. Acute meningitis. In: Mandell, Douglas and Bennett's Principles and Practice of Infectious Diseases. Eight Edition, 2015. p. 201-4.

3. van de Beek D, Brouwer MC, Thwaites GE, Tunkel AR. Advances in treatment of bacterial meningitis. Lancet. 2012:380(9854):1693-702. https://doi.org/10.1016/S01406736(12)61186-6 PMID: 23141618

4. Centers for Disease Control and Prevention (CDC). Effects of new penicillin susceptibility breakpoints for Streptococcus pneumoniae--United States, 2006-2007. MMWR Morb Mortal Wkly Rep. 2008;57(50):1353-5. PMID: 19092758

5. Marton A, Gulyas M, Munoz R, Tomasz A. Extremely high incidence of antibiotic resistance in clinical isolates of Streptococcus pneumoniae in Hungary. J Infect Dis. 1991;163(3):542-8. https://doi.org/10.1093/infdis/163.3.542 PMID: 1995728

6. Whitney CG, Farley MM, Hadler J, Harrison LH, Lexau C, Reingold $A$, et al. Increasing prevalence of multidrugresistant Streptococcus pneumoniae in the United States. N Engl J Med. 2000;343(26):1917-24. https://doi.org/10.1056/ NEJM200012283432603 PMID: 11136262

7. Ubukata K, Chiba N, Hasegawa K, Kobayashi R, Iwata $\mathrm{S}$, Sunakawa K. Antibiotic susceptibility in relation to penicillin-binding protein genes and serotype distribution of Streptococcus pneumoniae strains responsible for meningitis in Japan, 1999 to 2002. Antimicrob Agents Chemother. 2004;48(5):1488-94. https://doi.org/10.1128/AAC.48.5.1488 1494.2004 PMID: 15105095

8. Leggiadro RJ. Penicillin- and cephalosporin-resistant Streptococcus pneumoniae: an emerging microbial threat. Pediatrics. 1994;93(3):500-3. PMID: 8115213

9. París MM, Ramilo O, McCracken GH Jr. Management of meningitis caused by penicillin-resistant Streptococcus pneumoniae. Antimicrob Agents Chemother. 1995;39(10):2171 5. https://doi.org/10.1128/AAC.39.10.2171 PMID: 8619561

10. Tunkel AR, Hartman BJ, Kaplan SL, Kaufman BA, Roos KL, Scheld WM, et al. Practice guidelines for the management of bacterial meningitis. Clin Infect Dis. 2004;39(9):1267-84. https://doi.org/10.1086/425368 PMID: 15494903

11. Waisbourd-Zinman O, Bilavsky E, Tirosh N, Samra Z, Amir J. Penicillin and ceftriaxone susceptibility of Streptococcus pneumoniae isolated from cerebrospinal fluid of children with meningitis hospitalized in a tertiary hospital in Israel. Isr Med Assoc J. 2010;12(4):225-8. PMID: 20803882

12. van de Beek D, Cabellos C, Dzupova O, Esposito S, Klein M, Kloek AT, et al. ESCMID guideline: diagnosis and treatment of acute bacterial meningitis. Clin Microbiol Infect. 2016;22(Suppl 3):S37-62. https://doi.org/10.1016/j.cmi.2016.01.007 PMID: 27062097

13. John CC. Treatment failure with use of a third-generation cephalosporin for penicillin-resistant pneumococcal meningitis: case report and review. Clin Infect Dis. 1994;18(2):188-93. https://doi.org/10.1093/clinids/18.2.188 PMID: 8161625

14. Karlowsky JA, Thornsberry C, Jones ME, Evangelista AT, Critchley IA, Sahm DF, et al. Factors associated with relative rates of antimicrobial resistance among Streptococcus pneumoniae in the United States: results from the 
TRUST Surveillance Program (1998-2002). Clin Infect Dis. 2003;36(8):963-70. https://doi.org/10.1086/374052 PMID: 12684907

15. Cantón R, Morosini MI. Emergence and spread of antibiotic resistance following exposure to antibiotics. FEMS Microbiol Rev. 2011;35(5):977-91. https://doi.org/10.1111/j.15746976.2011.00295.x PMID: 21722146

16. Ben-Shimol S, Greenberg D, Givon-Lavi N, Schlesinger Y, Somekh E, Aviner S, et al. Early impact of sequential introduction of 7-valent and 13-valent pneumococcal conjugate vaccine on IPD in Israeli children 55 years: an active prospective nationwide surveillance. Vaccine. 2014;32(27):3452-9. https:// doi.org/10.1016/j.vaccine.2014.03.065 PMID: 24690148

17. Ben-Shimol S, Greenberg D, Givon-Lavi N, Schlesinger Y, Miron D, Aviner S, et al. Impact of $\mathrm{PCV}_{7} / \mathrm{PCV}_{13}$ introduction on invasive pneumococcal disease (IPD) in young children: Comparison between meningitis and non-meningitis IPD. Vaccine. 2016;34(38):4543-50. https://doi.org/10.1016/j. vaccine.2016.07.038 PMID: 27475471

18. Hsu HE, Shutt KA, Moore MR, Beall BW, Bennett NM, Craig AS, et al. Effect of pneumococcal conjugate vaccine on pneumococcal meningitis. N Engl J Med. 2009;360(3):244-56. https://doi.org/10.1056/NEJMoa0800836 PMID: 19144940

19. Richter SS, Diekema DJ, Heilmann KP, Dohrn CL, Riahi F, Doern GV. Changes in pneumococcal serotypes and antimicrobial resistance after introduction of the 13 -valent conjugate vaccine in the United States. Antimicrob Agents Chemother. 2014;58(11):6484-9. https://doi.org/10.1128/AAC.03344-14 PMID: 25136018

20. Weinberger DM, Malley R, Lipsitch M. Serotype replacement in disease after pneumococcal vaccination. Lancet. 2011;378(9807):1962-73. https://doi.org/10.1016/So1406736(10)62225-8 PMID: 21492929

21. Statistical Abstracts of Israel. 2014. No. 65. Central Bureau of Statistics. Jerusalem: State of Israel, 2014.

22. Ben-Shimol S, Greenberg D, Givon-Lavi N, Elias N, Glikman D, Rubinstein $U$, et al. Rapid reduction in invasive pneumococcal disease after introduction of $\mathrm{PCV}_{7}$ into the National Immunization Plan in Israel. Vaccine. 2012;30(46):66007. https://doi.org/10.1016/j.vaccine.2012.08.012 PMID 22939907

23. Ben-Shimol S, Givon-Lavi N, Grisaru-Soen G, Megged O, Greenberg D, Dagan R. Comparative incidence dynamics and serotypes of meningitis, bacteremic pneumonia and otherIPD in young children in the PCV era: Insights from Israeli surveillance studies. Vaccine. 2018;36(36):5477-84. https:// doi.org/10.1016/j.vaccine.2017.05.059 PMID: 28579230

24. Dagan R, Klugman KP. Impact of conjugate pneumococcal vaccines on antibiotic resistance. Lancet Infect Dis. 2008;8(12):785-95. https://doi.org/10.1016/S14733099(08)70281-0 PMID: 19022193

25. Kyaw MH, Lynfield R, Schaffner W, Craig AS, Hadler J, Reingold $A$, et al. Effect of introduction of the pneumococcal conjugate vaccine on drug-resistant Streptococcus pneumoniae. N Engl J Med. 2006;354(14):1455-63. https://doi.org/10.1056/ NEJMoa051642 PMID: 16598044

26. Fenoll A, Granizo IJ, Aguilar L, Giménez MJ, Aragoneses-Fenoll L, Hanquet G, et al. Temporal trends of invasive Streptococcus pneumoniae serotypes and antimicrobial resistance patterns in Spain from 1979 to 2007. J Clin Microbiol. 2009;47(4):1012-20. https://doi.org/10.1128/JCM.01454-08 PMID: 19225097

27. Deng X, Church D, Vanderkooi OG, Low DE, Pillai DR. Streptococcus pneumoniae infection: a Canadian perspective. Expert Rev Anti Infect Ther. 2013;11(8):781-91. https://doi.org/ 10.1586/14787210.2013.814831 PMID: 23977934

28. Dagan R, Barkai G, Givon-Lavi N, Sharf M, Vardy D, Cohen T, et al. Seasonality of antibiotic-resistant S. pneumoniae causing acute otitis media: A clue for antibiotic restriction policy? J Infect Dis. 2008;197(8):1094-102. https://doi. org/10.1086/528995 PMID: 18419528

29. Greenberg D, Givon-Lavi N, Sharf AZ, Vardy D, Dagan R. The association between antibiotic use in the community and nasopharyngeal carriage of antibiotic-resistant Streptococcus pneumoniae in Bedouin children. Pediatr Infect Dis J. 2008;27(9):776-82. https://doi.org/10.1097/ INF.obo13e3181715184 PMID: 18645545

30. Dagan R, Danino D, Ben-Shimol S, Greenberg D, Sharf A, GivonLavi N. Antibiotic prescription rates in children $<24$ months old following $P_{7} V_{7} / P V_{13}$ sequential implementation. The 55 th Anuual Meeting of the Infectious Diseases Society of America (IDSA). San Diego, CA, October 2017.

31. Tin Tin Htar M, Madhava H, Balmer P, Christopoulou D, Menegas D, Bonnet $\mathrm{E}$. A review of the impact of pneumococcal polysaccharide conjugate vaccine (7-valent) on pneumococcal meningitis. Adv Ther. 2013;30(8):748-62. https://doi. org/10.1007/s12325-013-0051-2 PMID: 24000099
32. Olarte L, Barson WJ, Barson RM, Lin PL, Romero JR, Tan TQ, et al. Impact of the 13-Valent Pneumococcal Conjugate Vaccine on Pneumococcal Meningitis in US Children. Clin Infect Dis. 2015;61(5):767-75. https://doi.org/10.1093/cid/civ368 PMID: 25972022

33. Jayasinghe S, Menzies R, Chiu C, Toms C, Blyth CC, Krause $\mathrm{V}$, et al. Long-term Impact of a " 3 + o" Schedule for 7 - and 13-Valent Pneumococcal Conjugate Vaccines on Invasive Pneumococcal Disease in Australia, 2002-2014. Clin Infect Dis. 2017;64(2):175-83. https://doi.org/10.1093/cid/ciw720 PMID: 27986682

34. Alari A, Chaussade H, Domenech De Cellès $M$, Le Fouler L, Varon E, Opatowski L, et al. Impact of pneumococcal conjugate vaccines on pneumococcal meningitis cases in France between 2001 and 2014: a time series analysis. BMC Med. 2016;14(1):211. https://doi.org/10.1186/s12916-016-0755-7 PMID: 27998266

35. Huang SS, Hinrichsen VL, Stevenson AE, Rifas-Shiman SL, Kleinman K, Pelton SI, et al. Continued impact of pneumococcal conjugate vaccine on carriage in young children. Pediatrics. 2009;124(1):e1-11. https://doi.org/10.1542/peds.2008-3099 PMID: 19564254

\section{License and copyright}

This is an open-access article distributed under the terms of the Creative Commons Attribution (CC BY 4.0) Licence. You may share and adapt the material, but must give appropriate credit to the source, provide a link to the licence, and indicate if changes were made.

This article is copyright of the authors or their affiliated institutions, 2018. 